\title{
Incorporation of Hybrid Crystalline Microporous Materials in Mixed-Matrix Membranes for Gas Separations
}

\author{
I.H. Musselman, E.V. Perez, M.J.C. Ordoñez, K.J. Balkus, Jr., and J.P. Ferraris \\ Department of Chemistry and Alan G. MacDiarmid NanoTech Institute, The University of Texas at \\ Dallas, Richardson, TX 75080
}

Large scale $\mathrm{CH}_{4} / \mathrm{CO}_{2}, \mathrm{H}_{2} / \mathrm{CO}_{2}, \mathrm{CH}_{4} / \mathrm{N}_{2}$, and $\mathrm{O}_{2}, \mathrm{~N}_{2}$ gas separations rely on energy intensive processes like distillation, condensation, and stripping. Although these processes are effective, they are expensive to operate. Membranes were developed in an attempt to lower the operational costs of gas separations and they have a smaller footprint and are scalable. From the variety of membranes available, we have focused on polymer-based mixed-matrix membranes (MMMs) for gas separations [1]. These types of membranes combine the processability of polymers with the separation properties of inorganic additives. In addition, they are less expensive and mechanically more robust than inorganic membranes (e.g., metallic, zeolite, ceramic, and carbon membranes), making them ideal materials for large-scale gas separations. However, the limited compatibility of inorganic additives with polymer matrices introduces non-selective voids in MMMs that limit the loading of the additives [2]. To mitigate this problem, metal-organic framework [MOF-5 (1.00 nm pore size), $\mathrm{Cu}-\mathrm{MOF}$ (0.95 nm pore size)], metal organic polyhedra [MOP-18 (1.5 nm pore size)], and zeolitic imidazolate framework [ZIF-8 $(1.10 \mathrm{~nm}$ pore size)] nanocrystals [3,4] (Fig. 1) were synthesized and mixed with the polyimide Matrimid ${ }^{\circledR}[5,6]$. These hybrid frameworks coordinate metals to organic linkers to build uniform pores. The sizes of the pores and their apertures can be adjusted to sieve gas molecules from mixtures. The presence of organic linkers in these materials makes them more compatible with polymer matrices, allowing for high loadings of up to $80 \%(\mathrm{w} / \mathrm{w})$ in the MMMs. For example, Cu-MOF improves gas solubility while ZIF-8 (0.34 nm pore aperture) causes molecular sieving of the gas molecules. Using this strategy, we have demonstrated that the separation properties of MMMs can be improved while maintaining their mechanical strength $[5,6]$.

SEM images of the Matrimid ${ }^{\circledR}$-based MMMs containing $80 \%$ (w/w) Cu-MOF (Fig. 2b) showed that the nanocrystals were uniformly dispersed in the polymer. Polymer veins and plastic deformation of the matrix were not observed indicating good wetting of the nanocrystals by the polymer as well as reduced chain rigidification. Gas permeation experiments showed that the $\mathrm{CH}_{4} / \mathrm{N}_{2}$ selectivity increased from 1.35 for Matrimid ${ }^{\circledR}$ to 2.05 for the $80 \%(\mathrm{w} / \mathrm{w}) \mathrm{MMM}$, with a simultaneous increase in $\mathrm{CH}_{4}$ permeability from 0.22 to 1650 Barrers. The solubilization of MOP-18 at the molecular level (Fig. 2c, AFM insert) resulted in MMMs with superior additive dispersion (Fig. 2c). The solubility of MOP-18 in the casting solvent is only achieved when the linker is functionalized with alkyl chains. However, the MOP-18/polymer interaction becomes critical for its dispersion in the polymer matrix when the solvent is evaporated.

The dispersion of ZIF-8 nanocrystals in Matrimid ${ }^{\circledR}$ (Fig. 2d) resulted in MMMs that exhibited increased $\mathrm{H}_{2} / \mathrm{CO}_{2}$ separation from 2.60 for Matrimid ${ }^{\circledR}$ to 7.00 for the $60 \%$ (w/w) ZIF-8 loading. SEM images of the cross-sections reveal the formation of polymer veins around the nanocrystals, suggesting the rigidification of polymer chains at the interface. As the loading of ZIF-8 approached $50 \%(\mathrm{w} / \mathrm{w})$, the separation properties of the MMM shifted from a polymer dominated to an additive driven process. 
References

[1] W.J. Koros and R. Mahajan, J. Membr. Sci. 175 (2000) 181.

[2] S. Husain and W.J. Koros, J. Membr. Sci. 288 (2007) 195.

[3] M. Eddaoudi et al., Science 295 (2002) 469.

[4] K.S. Park et al., Proc. Nat. Acad. Sci. 103 (2006) 10186.

[5] E.V. Perez et al., J. Membr. Sci. 328 (2009) 165.

[6] M.J.C. Ordoñez et al., J. Membr. Sci., in revision.

[7] Research supported by the U. S. Department of Energy, contracts: DE-FG26-04NT42173, DENT0007636, and DE-FE0001293 and the National Science Foundation grant No. 0933563.
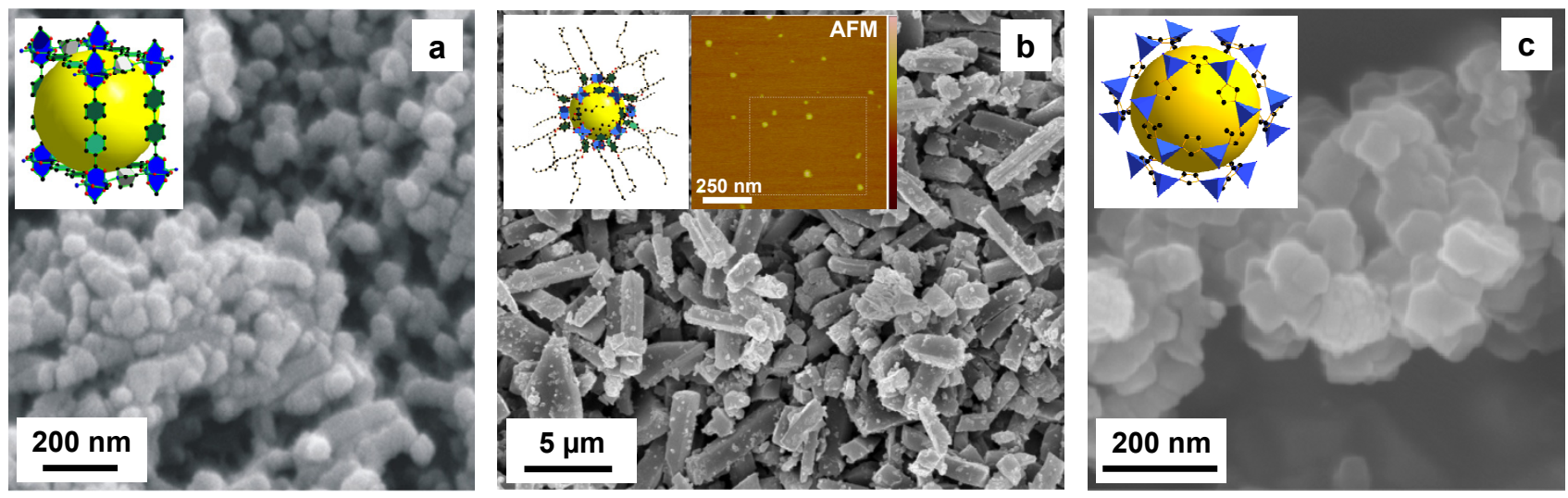

FIG. 1. Metal-organic frameworks used for the preparation of MMMs for gas separations (inserts show crystal structures): a) Cu-MOF, b) MOP-18, insert shows an AFM image of MOP-18 on mica, $\mathrm{z}$-scale $=5 \mathrm{~nm}, \mathrm{c}) \mathrm{ZIF}-8$.
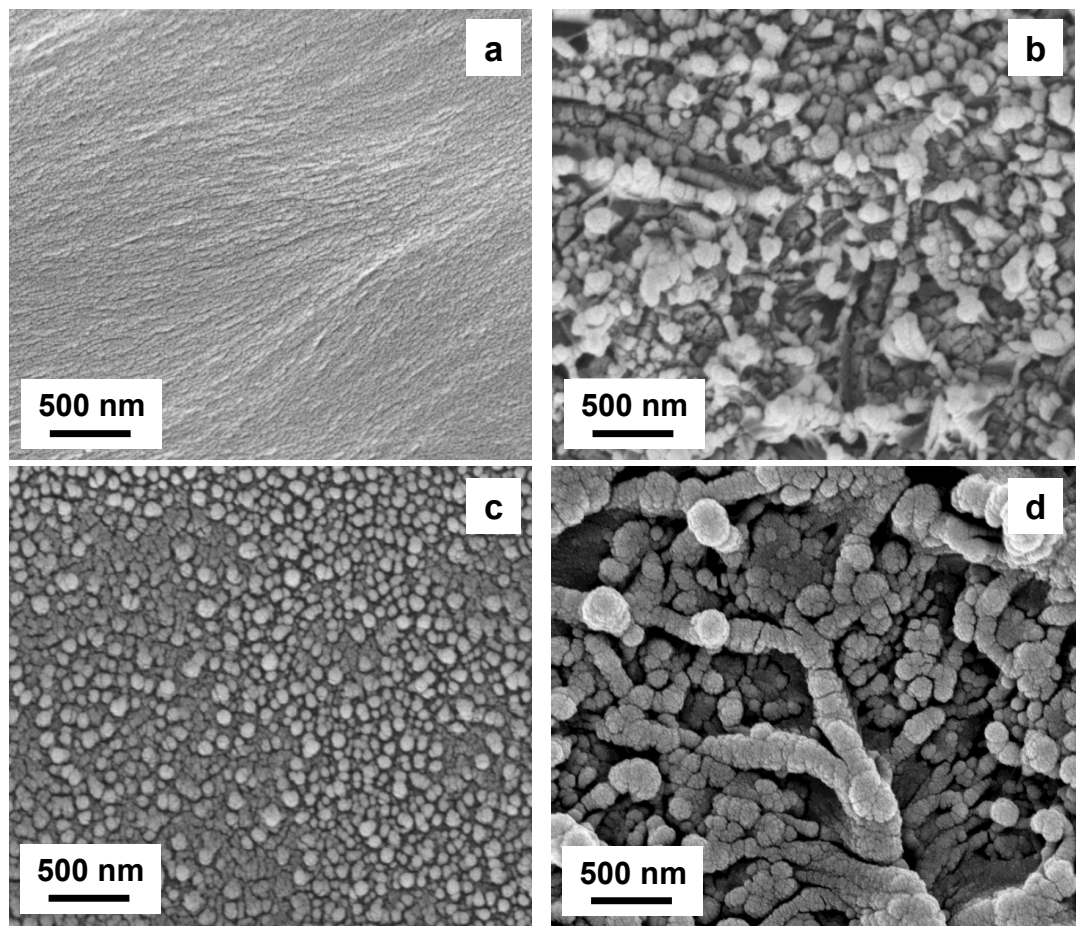

FIG. 2. SEM images of the cross-sections of MOFs and ZIFs in MMMs: a) Matrimid, b) 80\% (w/w) Cu-MOF, c) $80 \%$ (w/w) MOP-18, and d) 50\% (w/w) ZIF-8. 